\title{
Nonclassical states of light with a smooth $P$ function
}

\author{
François Damanet ${ }^{1,2}$, Jonas Kübler ${ }^{3}$, John Martin ${ }^{2}$, and Daniel Braun ${ }^{3}$ \\ 1 Department of Physics and SUPA, University of Strathclyde, Glasgow G4 0NG, United Kingdom. \\ 2 Institut de Physique Nucléaire, Atomique et de Spectroscopie, \\ CESAM, Université de Liège, Bâtiment B15, B - 4000 Liège, Belgium. \\ 3 Institut für theoretische Physik, Universität Tübingen, 72076 Tübingen, Germany.
}

\begin{abstract}
There is a common understanding in quantum optics that nonclassical states of light are states that do not have a positive semidefinite and sufficiently regular Glauber-Sudarshan $P$ function. Almost all known nonclassical states have $P$ functions that are highly irregular which makes working with them difficult and direct experimental reconstruction impossible. Here we introduce classes of nonclassical states with regular, non-positive-definite $P$ functions. They are constructed by "puncturing" regular smooth positive $P$ functions with negative Dirac-delta peaks, or other sufficiently narrow smooth negative functions. We determine the parameter ranges for which such punctures are possible without losing the positivity of the state, the regimes yielding antibunching of light, and the expressions of the Wigner functions for all investigated punctured states. Finally, we propose some possible experimental realizations of such states.
\end{abstract}

\section{INTRODUCTION}

Creating and characterizing nonclassical states of light are two tasks of substantial interest in a wide range of applications [1, 2], such as sub-shot-noise measurements [35], high resolution imaging [6 9], light source and detector calibration [10 12, quantum cryptography [13, 14, or quantum information processing [15].

In quantum optics, there is wide-spread agreement that the most classical pure states of light are coherent states $|\alpha\rangle$. These are eigenstates with complex eigenvalue $\alpha$ of the annihilation operator $a$ of the mode of the light-field under consideration, $a|\alpha\rangle=\alpha|\alpha\rangle$, and are characterized by the minimal uncertainty product of the quadratures $X=\left(a+a^{\dagger}\right) / \sqrt{2}$ and $Y=\left(a-a^{\dagger}\right) /(i \sqrt{2})$. In the case of a mechanical harmonic oscillator the quadratures correspond simply to phase space coordinates $x$ and $p$ in suitable units. Hence, a coherent state resembles most closely the notion of a classical phase space point located at the mean position of the quadratures. The label $\alpha \in \mathbb{C}$ determines these mean values as $\langle\alpha|X| \alpha\rangle=\sqrt{2} \operatorname{Re}(\alpha)$ and $\langle\alpha|Y| \alpha\rangle=\sqrt{2} \operatorname{Im}(\alpha)$. Coherent states of light, which can be produced by lasers operated far above the threshold [16], retain the property of minimal uncertainty $\Delta X \Delta Y=1 / 2$, where $\Delta X=\sqrt{\left\langle X^{2}\right\rangle-\langle X\rangle^{2}}$ is the standard deviation, under the dynamics of the harmonic oscillator underlying each mode of the electromagnetic field. Mixing classically coherent states (i.e. choosing randomly such states according to a classical probability distribution) should not increase their quantumness. Hence, (possibly mixed) quantum states given by a density operator $\rho$ as

$$
\rho=\int P(\alpha)|\alpha\rangle\langle\alpha| d^{2} \alpha
$$

with a positive $P$ function are considered "classical states" in quantum optics. While it may appear that (1) describes only states that are "diagonal" in a coherent state basis, it was shown by Sudarshan [17 that in fact all possible quantum states $\rho$ of the light-field (quantum or classical) can be represented by (1). This is possible due to the fact that coherent states are overcomplete. The price to pay, however, is that in general $P(\alpha)$ is not a smooth function, but a functional. Obviously, indeed, even a coherent state $\left|\alpha_{0}\right\rangle$ is already represented by a functional, namely a Dirac-delta peak, $P(\alpha)=\delta\left(\alpha-\alpha_{0}\right)$. Such a level of singularity represents the "boundary of acceptable irregular behavior" of the $P$ function for a classical state. Much worse singularities arise for example from simple Fock states $|n\rangle$, i.e. energy eigenstates of the mode, $a^{\dagger} a|n\rangle=n|n\rangle$, for which the $P$ function is given by the $n$-th derivative of a delta-function [18].

It would be desirable that one could directly reconstruct the $P$ function and in this way show the nonclassicality of states that are genuinely quantum. However, in the case of highly non-regular $P$ functions this is impossible. One way out is to apply filters that do not enhance the negativity of the characteristic function, i.e. the Fourier transform of $P(\alpha)[19] 22]$.

Also for the ease of theoretical work one would like to have classes of $P$ functions that are regular and smooth, but represent genuine quantumness due to the fact that they become negative [23]. One class of such states are single-photon added thermal states [20, i.e. states of the form $\rho=\mathcal{N} a^{\dagger} \rho_{T} a$, where $\rho_{T}$ represents a thermal state and $\mathcal{N}$ is a normalization factor. The $P$ function of this state is given by the product of a linear function of $|\alpha|^{2}$ and the Gaussian of the thermal state (see Eq. (4.36) in [18]). But as Agarwal writes (p.84 in [18]): "Here we perhaps have the unique case where $P(\alpha)$ exists but is negative. Most known cases of nonclassical $P$ involve $P$ functions which do not even exist."

In this paper, we introduce novel whole classes of such genuinely-quantum states with nevertheless smooth $P$ functions (i.e. exhibiting at most singularities in the form of Dirac $\delta$-functions). The recipe for doing so is very simple: Start with a classical quantum state with 
a smooth positive $P$ function, and then superpose some narrow, negative peaks. Altogether, one must guarantee, of course, that the density matrix $\rho$ remains a semidefinite positive operator. Finding parameter regimes where this is the case constitutes the main technical difficulty for constructing such states. Obviously, this general recipe allows for an infinity of possibilities, starting from the choice of the smooth original $P$ function, over the number and form of the negative peaks superposed, to their amplitudes and positions. We start with the simplest situation of the rotationally symmetric Gaussian $P$ function centered at $|\alpha|=0$ of a thermal state, "punctured" with a single negative delta-peak whose position and amplitude we can vary. Only the radial position is relevant here, and we have herewith already three parameters, exemplifying the wide range of tunability of punctured states.

The paper is organized as follows. In Section II, we introduce the "punctured states", their general properties, the conditions for positive definiteness of their density matrix representation and the conditions yielding nonclassical states. In Section III, we investigate the particular simple case of a thermal state punctured by a single delta-peak. In Section IV, we study the case of a squeezed thermal state punctured by a delta-peak. In Section V, we generalize our results by considering a thermal state punctured by a Gaussian peak of finite width. In each Section, we provide the expression of the Wigner function, calculate the second-order correlation function and identify the regimes of parameters yielding antibunching of light, i.e. sub-Poissonian statistics of the photon counting. In Section VI, we discuss some possibilities of experimentally creating some of these states. Finally, in Section VII, we give a conclusion of our work.

\section{DEFINITION, GENERAL PROPERTIES, POSITIVITY AND NONCLASSICALITY CONDITIONS}

\section{A. General punctured states}

Let $\rho_{\mathrm{cl}}$ represent a classical state, that is a state as defined in (1) with a smooth non-negative $P$ function $P_{\mathrm{cl}}(\alpha) \geqslant 0$. We define a "punctured $\rho_{\mathrm{cl}}$ state" as a state (1) with a $P$ function of the form

$$
P(\alpha)=\mathcal{N}\left[P_{\mathrm{cl}}(\alpha)-\sum_{i=1}^{N} w_{i} \pi_{i}\left(\alpha-\alpha_{i}\right)\right],
$$

where $\mathcal{N}$ is a normalization constant, the $w_{i}$ are positive coefficients representing the weights of the different punctures $i(i=1, \ldots, N)$, and $\pi_{i}(\alpha)$ are positive functions on the complex plane that determine their shape. We take them as normalized according to

$$
\int \pi_{i}(\alpha) d^{2} \alpha=1 \quad \forall i=1, \ldots, N
$$

If the weights are chosen such that the $P$ function (2) becomes negative for some $\alpha$ and that the resulting state $\rho$ is a positive semidefinite operator with unit trace, it follows immediately that the state is nonclassical. Furthermore, the $P$ function remains "well-behaved" (i.e. sufficiently regular), if $\pi_{i}(\alpha)$ are well-behaved, as we assumed $P_{\mathrm{cl}}(\alpha)$ smooth. In agreement with the common understanding that positive $P$ functions corresponding to simple Dirac- $\delta$ peaks are still accepted in the class of "well-behaved" functions because they represent coherent states, we consider in the following sections Dirac $\delta(\alpha)$ functions as worst singularities for the punctures $\pi_{i}(\alpha)$. This is the also the simplest case, as it does not contain any further free parameter.

\section{B. Conditions for (semi)positive definiteness}

For a given choice of the functions $\pi_{i}(\alpha)$ for the punctures, positivity of the state will depend on the position of the $\alpha_{i} \in \mathbb{C}$ and the weights $w_{i}$. Our task is hence to find the allowed parameter ranges for $\left\{w_{i}, \alpha_{i}\right\}$ such that $\rho \geqslant 0$, i.e. $\rho$ is a positive semidefinite operator. The normalization factor $\mathcal{N}$ ensures that $\int P(\alpha) d^{2} \alpha=1$, i.e.

$$
\mathcal{N}=\left(1-\sum_{i=1}^{N} w_{i}\right)^{-1}
$$

Since $\mathcal{N}$ does not influence the positivity, we will often skip it.

It is clear that in general the condition for any single parameter will depend on the values of all the others. This can be seen immediately from the general necessary and sufficient condition of positive semidefiniteness, $\langle\psi|\rho| \psi\rangle \geqslant 0 \forall|\psi\rangle \in \mathcal{H} \equiv L^{2}(\mathbb{C})$, which for the state 1 with a $P$ function of the form (2) reads

$$
\int\left(P_{\mathrm{cl}}(\alpha)-\sum_{i} w_{i} \pi_{i}\left(\alpha-\alpha_{i}\right)\right)|\langle\psi \mid \alpha\rangle|^{2} d^{2} \alpha \geqslant 0 \forall|\psi\rangle \in \mathcal{H} .
$$

To go beyond this general criterion, we first review briefly a few necessary and sufficient conditions for positivity of a linear operator.

Necessary and sufficient condition $(\mathcal{N S C})$. A necessary and sufficient condition $(\mathcal{N S C})$ for a hermitian matrix $\rho$ to be semi-positive definite $(\rho \geqslant 0)$ is that all its eigenvalues are non-negative. While the eigenvalues of an infinite dimensional matrix are generally not readily accessible, the condition nevertheless allows for a numerical approach. Indeed, the density operator (1) can be expressed in the Fock state basis $\left\{|n\rangle: n \in \mathbb{N}_{0}\right\}$ using the expansion of coherent states

$$
|\alpha\rangle=e^{-\frac{|\alpha|^{2}}{2}} \sum_{n=0}^{\infty} \frac{\alpha^{n}}{\sqrt{n !}}|n\rangle .
$$

Then, by introducing a cut-off dimension in Hilbert space of sufficiently high excitation $n_{\max }$, a finite dimensional 
matrix representation can be obtained and one can check numerically that when increasing the cut-off, the lowest eigenvalue converges to a value that is sufficiently far from zero for judging the positivity of the state.

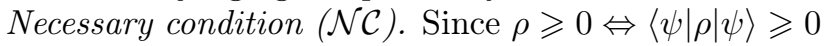
for all states $|\psi\rangle$, a necessary condition $(\mathcal{N C})$ for $\rho \geqslant 0$ is that all diagonal elements $\rho_{\phi \phi} \equiv\langle\phi|\rho| \phi\rangle$ of the density matrix are non-negative for a given class of states $|\phi\rangle \in$ $\mathcal{K} \subset \mathcal{H}$ where $\mathcal{K}$ is a subset of $\mathcal{H}$, i.e.

$$
\rho_{\phi \phi} \equiv\langle\phi|\rho| \phi\rangle \geqslant 0, \forall|\phi\rangle \in \mathcal{K} \Leftarrow \rho \geqslant 0 .
$$

Sufficient condition $(\mathcal{S C})$. A sufficient condition $(\mathcal{S C})$ for positivity can be found from Gershgorin's Circle Theorem. Consider first the case of finite dimensional matrices $A$. The theorem then says [24]: "Every eigenvalue of a complex square matrix $A$ of coefficients $\left\{a_{i j}\right\}$ lies within at least one of the Gershgorin disks $D\left(a_{i i}, R_{i}\right)$ centred at $a_{i i}$ with radii $R_{i}=\sum_{j \neq i}\left|a_{i j}\right|$ or $R_{i}=\sum_{j \neq i}\left|a_{j i}\right|$." An interpretation of this theorem is that if the off-diagonal elements of $A$ have small norms compared to its diagonal elements, its eigenvalues cannot be far from the values of the diagonal elements. A matrix is called (strictly) diagonally dominant if $\left|a_{i i}\right| \geqslant R_{i}\left(\left|a_{i i}\right|>R_{i}\right)$ for all $i$. This leads to the following sufficient condition: a hermitian (strictly) diagonally dominant matrix with real nonnegative diagonal entries is positive semidefinite (definite). Gershgorin's Circle Theorem has been generalized to the case of infinite dimensional matrices in [25], where a proof was given for matrices of the space $L^{1}$. The proof for the space $L^{2}$ in which our operators live can be done analogously and leads to the same result, namely that the eigenvalues lie in $\cup_{i=1}^{\infty} D\left(a_{i i}, R_{i}\right)$ (just as in the case of a finite space). Hence, by expressing the density matrix in the Fock state basis, we obtain the sufficient condition $(\mathcal{S C})$

$$
\rho_{n n}-R_{n} \geqslant 0 \quad \forall|n\rangle \quad \Rightarrow \quad \rho \geqslant 0,
$$

where $\rho_{n n} \equiv\langle n|\rho| n\rangle$ and $R_{n}=\sum_{m \neq n}\left|\rho_{n m}\right|$.

\section{Conditions for nonclassicality}

There exist various nonclassicality criteria for radiation fields. In this work, we shall consider criteria based on the negativity of the $P$ function, the negativity of the Wigner function and the existence of antibunching [1].

Negativity of the $P$ function. A criterion for a state to be nonclassical is that its $P$ function takes negative values, so that it can no longer be interpreted as a probability distribution in phase space. For example, all physical $\delta$-punctured states are nonclassical according to this criterion. Other punctured states, however, could have a non-negative $P$ function in the whole complex plane, so that general punctured states are not necessarily nonclassical.

Negativity of the Wigner function. Negativity of the Wigner function is a widely used criterion for nonclassicality. This is largely due to the fact that the Wigner function can be directly measured (see e.g. [26 31]). Since the Wigner function $W(\alpha)$ is the convolution of the $P$ function with the vacuum state, i.e.

$$
W(\alpha)=\frac{2}{\pi} \int P(\beta) e^{-2|\alpha-\beta|^{2}} d^{2} \beta,
$$

the existence of negative values for the Wigner function implies the existence of negative values for the $P$ function, but the reverse is not true. Thus, the criterion based on negative values of the Wigner function detects fewer nonclassical states than the previous criterion. More specifically, it does not detect states with partly negative $P$ function but everywhere positive Wigner function. This explains why the definitions of punctured states based on the puncturing of $P$ functions rather than Wigner functions is more appropriate. Moreover, deltapuncturing of a Wigner function would be forbidden as Wigner functions are always bounded (see e.g. p. 73 in [32]).

Antibunching. Another standard criterion of nonclassicality is based on the second order correlation function

$$
g^{(2)}=\frac{\left\langle a^{\dagger} a^{\dagger} a a\right\rangle}{\left\langle a^{\dagger} a\right\rangle^{2}},
$$

where $\langle\cdot\rangle=\operatorname{Tr}(\cdot \rho)$, or, equivalently, on the Mandel $Q_{M}$ parameter 33 ]

$$
Q_{M}=\frac{(\Delta n)^{2}-\langle n\rangle}{\langle n\rangle}=\langle n\rangle\left(g^{(2)}-1\right),
$$

where $n=a^{\dagger} a$. A state is said to be nonclassical if $g^{(2)}<1$ (or, equivalently, $Q_{M}<0$ ), which corresponds to antibunching (sub-Poissonian statistics of photon counting that cannot be observed for mixtures of coherent states).

It is interesting to note the connection between classicality in terms of the $P$ function and in terms of antibunching, pointed out by Kimble et al. 34, and generalized by Vogel to space- and time-dependent correlations [35]: $g^{(2)}<1$ can exist if the $P$ function is negative somewhere. Conversely, if the $P$ function of a state is a valid probability density, then there is no antibunching, i.e. $g^{(2)} \geqslant 1$. Proof. This statement can be proved using Jensen's inequality. We use equation (5') in the original paper [36]. Let $P(\alpha)$ be a valid probability density defined over the complex numbers. Then $P_{r}(r)=\int_{0}^{2 \pi} r P\left(r e^{i \phi}\right) d \phi$ defines a valid probability density over the real numbers $r \in[0, \infty)$. Using Eq. (1), the second order correlation function 10 can be written as

$$
g^{(2)}=\frac{\int|\alpha|^{4} P(\alpha) d^{2} \alpha}{\left[\int|\alpha|^{2} P(\alpha) d^{2} \alpha\right]^{2}}=\frac{\int_{0}^{\infty} r^{4} P_{r}(r) d r}{\left[\int_{0}^{\infty} r^{2} P_{r}(r) d r\right]^{2}} .
$$

Defining the convex functions $f(r)=\varphi(r)=r^{2}$, we can rewrite the above as

$$
g^{(2)}=\frac{\int_{0}^{\infty} \varphi(f(r)) P_{r}(r) d r}{\varphi\left(\int_{0}^{\infty} f(r) P_{r}(r) d r\right)} .
$$


The condition $g^{(2)} \geqslant 1$ is then equivalent to

$$
\int_{0}^{\infty} \varphi(f(r)) P_{r}(r) d r \geqslant \varphi\left(\int_{0}^{\infty} f(r) P_{r}(r) d r\right),
$$

which is true by Jensen's inequality.

As a conclusion, for radiation field states, both the negativity of the Wigner function and antibunching imply that the $P$ function is not a valid probability density, while the reverse is not necessarily true.

In the following sections, we investigate the cases of various punctured states. In each case, we apply the conditions of positivity of and nonclassicality of $\rho$ to determine the physical nonclassical states. For the sake of simplicity, we restrict ourselves in the remainder of the paper to a single puncture by setting $N=1$ in Eq. (2).

\section{DELTA-PUNCTURED THERMAL STATES}

We define a $\delta$-punctured thermal state as a state determined through (1) and (2) with

$$
\begin{aligned}
& P_{\mathrm{cl}}(\alpha)=\frac{e^{-|\alpha|^{2} / \bar{n}}}{\pi \bar{n}}, \\
& \pi(\alpha)=\delta(\alpha),
\end{aligned}
$$

where $\bar{n}=\left(e^{\hbar \omega / k_{B} T}-1\right)^{-1}$ is the average number of thermal excitations at temperature $T$ of the radiation field with frequency $\omega$.

\section{A. Conditions for (semi)positive definiteness}

Necessary condition. A necessary condition for the positivity of $\rho$ can be obtained from the condition (7) with $\mathcal{K}$ the set of coherent states. It yields the following upper bound for the puncture weight $w_{1}$ :

$$
w_{1} \leqslant \frac{e^{-\left|\alpha_{1}\right|^{2} / \bar{n}}}{\bar{n}+1} \equiv w_{\max }^{\delta, \mathcal{N C}} .
$$

Proof. Let $|\gamma\rangle(\gamma \in \mathbb{C})$ denote a coherent state. The density matrix element $\rho_{\gamma \gamma} \equiv\langle\gamma|\rho| \gamma\rangle$ is then given by

$$
\rho_{\gamma \gamma}=\mathcal{N}\left[\frac{1}{\pi \bar{n}} \int e^{-\frac{|\alpha|^{2}}{\bar{n}}-|\alpha-\gamma|^{2}} d^{2} \alpha-w_{1} e^{-\left|\alpha_{1}-\gamma\right|^{2}}\right],
$$

where we used $|\langle\gamma \mid \alpha\rangle|^{2}=e^{-|\alpha-\gamma|^{2}}$. The condition that $\rho_{\gamma \gamma}$ must be non-negative for all $\gamma$ reads

$$
w_{1} \leqslant \frac{1}{\pi \bar{n}} \frac{\int e^{-\frac{|\alpha|^{2}}{\bar{n}}-|\alpha-\gamma|^{2}} d^{2} \alpha}{e^{-\left|\alpha_{1}-\gamma\right|^{2}}} \quad \forall \gamma \in \mathbb{C} .
$$

After integration, this yields the condition

$$
w_{1} \leqslant \frac{e^{-\left(\frac{1}{\bar{n}+1}\right)|\gamma|^{2}+\left|\alpha_{1}-\gamma\right|^{2}}}{\bar{n}+1} \quad \forall \gamma \in \mathbb{C} .
$$

The minimum over $\gamma$ of the rhs term is attained for $\gamma=$ $\left(\frac{\bar{n}+1}{\bar{n}}\right) \alpha_{1}$ and directly leads to the condition 16 .

Sufficient condition. In the Fock state basis, the Gershgorin disks $D\left(\rho_{n n}, R_{n}\right)$ have center $\rho_{n n} \equiv\langle n|\rho| n\rangle$ and radius $R_{n}$ given by

$$
\begin{aligned}
& \rho_{n n}=\frac{\bar{n}^{n}}{(\bar{n}+1)^{n+1}}-w_{1} e^{-\left|\alpha_{1}\right|^{2}} \frac{\left|\alpha_{1}\right|^{2 n}}{n !}, \\
& R_{n}=w_{1} e^{-\left|\alpha_{1}\right|^{2}} \frac{\left|\alpha_{1}\right|^{n}}{\sqrt{n !}} \sum_{\substack{m=0 \\
m \neq n}}^{+\infty} \frac{\left|\alpha_{1}\right|^{m}}{\sqrt{m !}} .
\end{aligned}
$$

The sufficient condition (8) then implies the following condition on $w_{1}$,

$$
w_{1}<\frac{e^{\left|\alpha_{1}\right|^{2}}}{\bar{n}+1} \frac{1}{f\left(\left|\alpha_{1}\right|\right)} \min _{n \in \mathbb{N}}\left\{\left(\frac{\frac{\bar{n}}{\bar{n}+1}}{\left|\alpha_{1}\right|}\right)^{n} \sqrt{n !}\right\} \equiv w_{\max }^{\delta, \mathcal{S C}}
$$

where

$$
f\left(\left|\alpha_{1}\right|\right)=\sum_{m=0}^{+\infty} \frac{\left|\alpha_{1}\right|^{m}}{\sqrt{m !}}
$$

The bound $w_{\max }^{\delta, \mathcal{S C}}$ depends only on the absolute value of $\alpha_{1}$, not on its phase. Also, we have $w_{\max }^{\delta, \mathcal{S C}}=0$ only if $\bar{n}=0$. The largest values of $w_{1}$ allowed by the bound lie in the region where $\left|\alpha_{1}\right|$ and $\bar{n}$ are small, which is shown in Fig. 1. Since $w_{\max }^{\delta, \mathcal{S C}}>0$ for all values of $\bar{n}>0$ and $\alpha_{1}$, this is an analytical proof that there always exist physical nonclassical $\delta$-punctured thermal states.

Tightness of $w_{\max }^{\delta, \mathcal{N C}}$. Computations based on the diagonalization of $\delta$-punctured thermal states and the application of $\mathcal{N S C}$ suggest that the bound $w_{\max }^{\delta, \mathcal{N C}}$ given in Eq. 16 is tight. This claim is further supported by the observation that the coherent state $\left|\left(\frac{\bar{n}+1}{\bar{n}}\right) \alpha_{1}\right\rangle$ from which the bound is derived is an eigenstate of $\rho$ with $w_{1}=w_{\max }^{\delta, \mathcal{N C}}$ with eigenvalue 0 . In Fig. 11 we display both $w_{\max }^{\delta, \mathcal{N}}$ and the value of $w_{1}$ that cancels the smallest eigenvalue of $\rho$. In our computations, the density matrix is expressed in the truncated Fock state basis $\left\{|0\rangle,|1\rangle, \ldots,\left|n_{\max }\right\rangle\right\}$ with a sufficiently large cut-off $n_{\max }$ in order ensure stable numerical results. An analytical proof of the tightness for a puncture at $\alpha_{1}=0$ will be provided in the context of Gaussian punctures (Sec. VA). Note that a delta-puncture at $\alpha=0$ with maximum weight $w_{\max }^{\delta, \mathcal{N C}}$ corresponds to the complete removal of the ground-state of the oscillator ("the vacuum" in quantum-field theory parlance) from the state. We call such states "vacuum-removed states" for short.

In summary, our results indicate that any $\delta$-punctured thermal state with $w_{1}$ satisfying Eq. 16 corresponds to a proper physical state. As can be seen in Fig. 1, the value of the bound of allowed weights $w_{1}$ decreases as $\left|\alpha_{1}\right|$ increases, showing that a thermal state cannot be punctured significantly far from its center. This feature is less pronounced as $\bar{n}$ increases. 


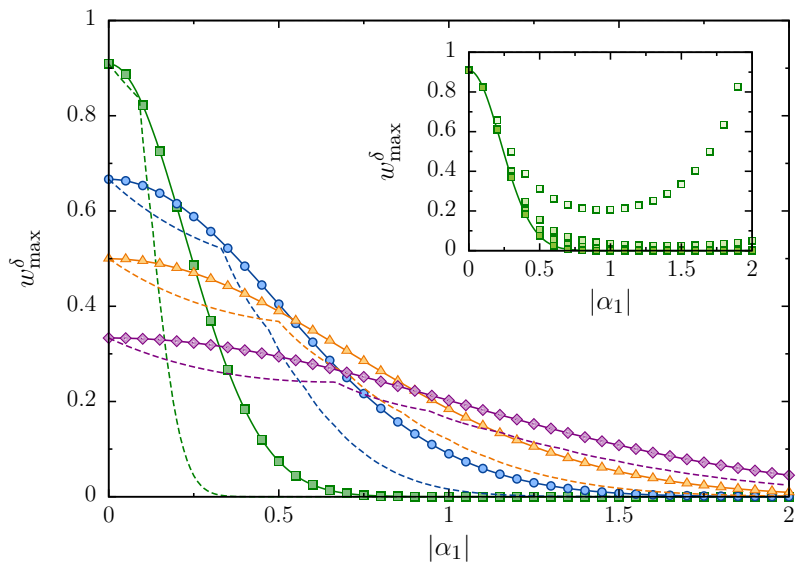

FIG. 1. Plot of the upper bounds $w_{\max }^{\delta, \mathcal{N C}}$ (Eq. 16, solid curves) and $w_{\max }^{\delta, \mathcal{S C}}$ (Eq. 22, dashed curves), and of $w_{\max }^{\delta}$, the $w_{1}$ which cancels the smallest eigenvalue of the density matrix, as a function of $\left|\alpha_{1}\right|$ for different $\bar{n}$ (green squares: $\bar{n}=0.1$, blue dots: $\bar{n}=0.5$, orange triangles: $\bar{n}=1$, and purple diamonds: $\bar{n}=2$ ). The eigenvalues are computed in a Fock space of dimension $n_{\max }=15$. Inset: convergence of $w_{\max }^{\delta}$ (green squares) with increasing cut-off dimension $n_{\max }$ $\left(n_{\max }=2,3,4\right.$ and 15 from top to bottom) for $\bar{n}=0.1$.

\section{B. Conditions for nonclassicality}

Negativity of the $P$ function. All $\delta$-punctured thermal states are nonclassical due to the presence of the infinite negative $\delta$-peak in the $P$ function.

Negativity of the Wigner function. The Wigner function of $\delta$-punctured thermal states follows from Eq. (9) and is given by

$$
W(\alpha)=\frac{2 \mathcal{N}}{\pi}\left(\frac{e^{-\frac{2|\alpha|^{2}}{1+2 \bar{n}}}}{1+2 \bar{n}}-w_{1} e^{-2\left|\alpha-\alpha_{1}\right|^{2}}\right) .
$$

It takes negative values for large enough puncture weight

$$
w_{1}>\frac{e^{-\left|\alpha_{1}\right|^{2} / \bar{n}}}{1+2 \bar{n}} .
$$

Such weights are still acceptable as long as they do not exceed the (larger) bound (16).

Antibunching. For $\delta$-punctured thermal states, the second-order correlation function 10 is given by

$$
g^{(2)}=\left(1-w_{1}\right) \frac{2 \bar{n}^{2}-w_{1}\left|\alpha_{1}\right|^{4}}{\left(\bar{n}-w_{1}\left|\alpha_{1}\right|^{2}\right)^{2}}
$$

For $w_{1} \rightarrow 0$, we recover the known value $g^{(2)}=2$ of the thermal state 37. In Fig. 2, we show a density plot of $g^{(2)}$ as a function of $\bar{n}$ and $\left|\alpha_{1}\right|$ for the maximal allowed value of the puncture weight $w_{1}$, i.e. the bound $(16)$. A whole region of parameter space corresponds to punctured states giving rise to antibunching $\left(g^{(2)}<1\right)$. Also, $g^{(2)}$ increases beyond 2 for $\bar{n} \simeq 0.1,\left|\alpha_{1}\right| \simeq 0.5$.

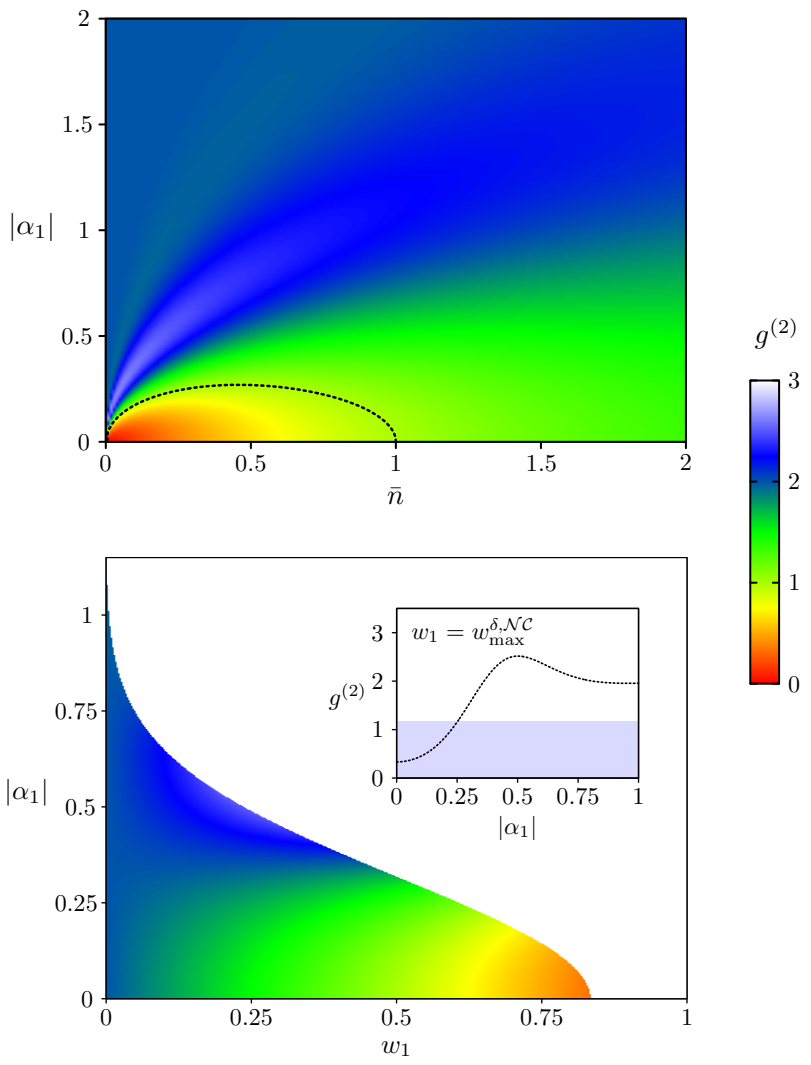

FIG. 2. Density plot of the second order correlation function $g^{(2)}$ [Eq. 26] for single $\delta$-punctured thermal states as a function of $\left|\alpha_{1}\right|$ and $\bar{n}$ for the maximal puncture weight $w_{1}=w_{\max }^{\delta, \mathcal{N C}}$ given by Eq. 16 (top) and as a function of $\left|\alpha_{1}\right|$ and $w_{1}$ for $\bar{n}=0.2$ (bottom). Only the range where $\rho \geqslant 0$ is shown. The black dashed curve in the top figure, along which $g^{(2)}=1$, delimits the sets of states displaying antibunching or not. The inset of the bottom figure represents $g^{(2)}$ as a function of $\alpha_{1}$ for the maximum weight $w_{1}=w_{\max }^{\delta, \mathcal{N C}}$.

\section{DELTA-PUNCTURED SQUEEZED THERMAL STATES}

To generalize the previous results, we now replace the thermal state by a squeezed thermal state [38, 39.

$$
\rho_{\mathrm{cl}}=\frac{1}{\pi \bar{n}} \int e^{-|\alpha|^{2} / \bar{n}} S(r)|\alpha\rangle\langle\alpha| S^{\dagger}(r) d^{2} \alpha,
$$

where $S(r)=\exp \left(-r\left[a^{\dagger 2}-a^{2}\right] / 2\right)$ is the squeezing operator and $r$ is the squeezing parameter, taken real for simplicity. We remind that the $P$ function of a state $\rho$ is given by the Fourier transform of its normal-ordered characteristic function $\chi(\beta)=\operatorname{tr}\left[\rho e^{i\left(\beta a^{\dagger}+\beta^{*} a\right)}\right]$. For the squeezed thermal state $\rho_{\mathrm{cl}}$ defined in Eq. (27), the normal-ordered characteristic function reads [38]

$$
\chi(\beta)=e^{-\bar{n}_{I} \beta_{R}^{2}-\bar{n}_{R} \beta_{I}^{2}},
$$


where $\beta_{R}$ and $\beta_{I}$ are the real and imaginary parts of $\beta \in \mathbb{C}$, and

$$
\begin{aligned}
& \bar{n}_{R}=e^{-2 r} \bar{n}-e^{-r} \sinh r, \\
& \bar{n}_{I}=e^{2 r} \bar{n}+e^{r} \sinh r .
\end{aligned}
$$

The Fourier transform of the characteristic function - and thus the $P$ function - exists only if both the coefficients $\bar{n}_{I}$ and $\bar{n}_{R}$ in front of $\beta_{R}^{2}$ and $\beta_{I}^{2}$ are positive, which imposes the condition

$$
e^{-2|r|} \bar{n}-e^{-|r|} \sinh |r|>0
$$

thereby limiting the range of allowed squeezing parameters $r \in \mathbb{R}$ for a given $\bar{n}$. The characteristic function of a squeezed thermal state being an asymmetric Gaussian distribution, the same goes for its $P$ function. Therefore, a $\delta$-punctured squeezed thermal state is a state determined through (1) and (2) with

$$
\begin{aligned}
& P_{\mathrm{cl}}(\alpha)=\frac{e^{-\left(\frac{\alpha_{R}^{2}}{\bar{n}_{R}}+\frac{\alpha_{I}^{2}}{\bar{n}_{I}}\right)}}{\pi \sqrt{\bar{n}_{R} \bar{n}_{I}}}, \\
& \pi(\alpha)=\delta(\alpha),
\end{aligned}
$$

where $\alpha_{R}$ and $\alpha_{I}$ are the real and imaginary parts of $\alpha \in \mathbb{C}$.

\section{A. Conditions for (semi)positive definiteness}

Necessary condition. Since $P_{\mathrm{cl}}(\alpha)$ is rotationally nonsymmetric, it is reasonable to assume that the necessary condition (7) will provide us with a tight bound for $w_{1}$ only if we consider states $|\phi\rangle \in \mathcal{K}$ with non-symmetric $P$-functions. By taking $\mathcal{K}$ to be the set of squeezed coherent states [38, 39, we obtain the following upper bound for the puncture weight $w_{1}$ :

$$
w_{1} \leqslant \frac{2 \sqrt{\bar{n}_{R} \bar{n}_{I}} e^{-\left(\frac{\alpha_{1 R}^{2}}{\bar{n}_{R}}+\frac{\alpha_{1 I}^{2}}{\bar{n}_{I}}\right)}}{\bar{n}_{R}+\bar{n}_{I}+2 \bar{n}_{R} \bar{n}_{I}} \equiv w_{\max , \mathrm{squ}}^{\delta, \mathcal{N C}} .
$$

When $\bar{n}_{R}=\bar{n}_{I}=\bar{n}$, this condition reduces to the condition (16), as expected.

Proof. Let us denote by $\left|\gamma, r^{\prime}\right\rangle$ a squeezed coherent state defined as [38, 39]

$$
\left|\gamma, r^{\prime}\right\rangle=D(\gamma) S\left(r^{\prime}\right)|0\rangle,
$$

where $D(\gamma)=\exp \left(\gamma a^{\dagger}-\gamma^{*} a\right)$ is the displacement operator and $S\left(r^{\prime}\right)$ the squeezing operator of parameter $r^{\prime}$, where the prime symbol distinguishes $r^{\prime}$ from $r$, this latter defining $\bar{n}_{R}$ and $\bar{n}_{I}$. The necessary condition (7) with $|\phi\rangle=\left|\gamma, r^{\prime}\right\rangle$ reads

$$
w_{1} \leqslant \frac{\int P_{\mathrm{cl}}(\alpha)\left|\left\langle\gamma, r^{\prime} \mid \alpha\right\rangle\right|^{2} d^{2} \alpha}{\left|\left\langle\gamma, r^{\prime} \mid \alpha_{1}\right\rangle\right|^{2}},
$$

where the modulus squared of the scalar product between a squeezed coherent state $\left|\gamma, r^{\prime}\right\rangle$ and a coherent state $|\alpha\rangle$ is given by [37]

$$
\begin{array}{r}
\left|\left\langle\gamma, r^{\prime} \mid \alpha\right\rangle\right|^{2}=\operatorname{sech}\left(r^{\prime}\right) e^{-\left(|\alpha|^{2}+|\gamma|^{2}\right)+\left(\alpha^{*} \gamma+\gamma^{*} \alpha\right) \operatorname{sech}\left(r^{\prime}\right)} \\
\times e^{-\operatorname{th}\left(r^{\prime}\right) \operatorname{Re}\left[\alpha^{2}-\gamma^{2}\right]}
\end{array}
$$

The minimum of the rhs term in Eq. (34) is obtained, after integration over $\alpha$, for

$$
\begin{aligned}
\gamma & =\frac{\bar{n}_{R}+\bar{n}_{I}+2 \bar{n}_{R} \bar{n}_{I}}{\sqrt{\left(\bar{n}_{R}-\bar{n}_{I}+2 \bar{n}_{R} \bar{n}_{I}\right)\left(\bar{n}_{I}-\bar{n}_{R}+2 \bar{n}_{R} \bar{n}_{I}\right)}} \alpha_{1}, \\
r^{\prime} & =\operatorname{arctanh}\left(\frac{\bar{n}_{R}-\bar{n}_{I}}{2 \bar{n}_{R} \bar{n}_{I}}\right)
\end{aligned}
$$

and leads to the necessary condition 32 .

Tightness of $w_{\max , \mathrm{squ}}^{\delta, \mathcal{N C}}$. Computations relying on the diagonalization of $\rho$ given by Eq. (1) with Eqs. (2) and (31) suggest that the bound $w_{\max , \text { squ }}^{\delta, \mathcal{N C}}$ given in Eq. (32) is tight. As for the case of thermal states, this claim is supported by the observation that the squeezed coherent state $\left|\gamma, r^{\prime}\right\rangle$ with $\gamma$ and $r^{\prime}$ given by Eq. (36) is an eigenstate of $\rho$ with eigenvalue 0 . Figure 3 shows $w_{\max , \text { squ }}^{\delta, \mathcal{N C}}$ and the value of $w_{1}$ that cancels the smallest eigenvalue of $\rho$. Numerical results converge to the bound as the cut-off dimension increases.

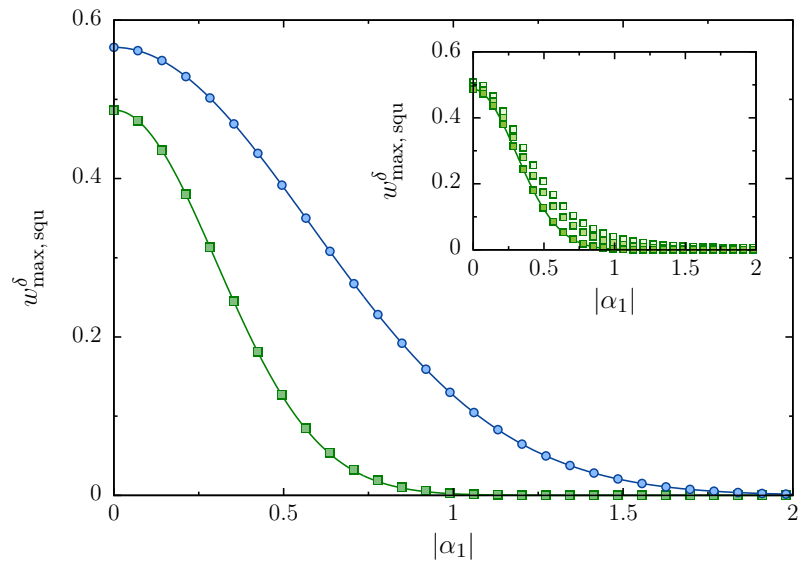

FIG. 3. Plot of the upper bound $w_{\max }^{\delta, \mathcal{N} \mathcal{C}}$ squ (solid curves) and of $w_{\max , \mathrm{squ}}^{\delta}$, the $w_{1}$ which cancels the smallest eigenvalue of the density matrix, as a function of $\left|\alpha_{1}\right|$ for $\bar{n}_{R}=1, \bar{n}_{I}=0.1$ (squares) and $\bar{n}_{I}=0.5$ (dots). The eigenvalues are computed in a Fock space of dimension $n_{\max }=25$. Inset: convergence of $w_{\max , \text { squ }}^{\delta}$ (green squares) with increasing cut-off dimension $n_{\max }\left(n_{\max }=6,8,25\right.$ from top to bottom $)$ for $\bar{n}_{I}=0.1$.

\section{B. Conditions for nonclassicality}

Negativity of the $P$ function. As for $\delta$-punctured thermal states, the presence of a $\delta$ peak always enforces nonclassicality. 
Negativity of the Wigner function. The Wigner function of a $\delta$-punctured squeezed thermal state reads

$$
W(\alpha)=\frac{2 \mathcal{N}}{\pi}\left(\frac{e^{-2\left(\frac{\alpha_{R}^{2}}{1+2 n_{R}}+\frac{\alpha_{I}^{2}}{1+2 n_{I}}\right)}}{1+2 \bar{n}}-w_{1} e^{-2\left|\alpha-\alpha_{1}\right|^{2}}\right),
$$

and takes negative values if the puncture weight satisfies

$$
w_{1}>\frac{e^{-\left(\frac{\alpha_{R}^{2}}{\bar{n}_{R}}+\frac{\alpha_{I}^{2}}{\bar{n}_{I}}\right)}}{1+2 \bar{n}},
$$

which is still acceptable as long as it does not exceed the (larger) bound (32).

Antibunching. For $\delta$-punctured squeezed thermal states, the second-order correlation function $(10)$ is given by

$$
g^{(2)}=\left(1-w_{1}\right) \frac{\frac{1}{4}\left(3 \bar{n}_{R}^{2}+2 \bar{n}_{R} \bar{n}_{I}+3 \bar{n}_{I}^{2}\right)-w_{1}\left|\alpha_{1}\right|^{4}}{\left(\frac{\bar{n}_{R}+\bar{n}_{I}}{2}-w_{1}\left|\alpha_{1}\right|^{2}\right)^{2}} .
$$

For $w_{1} \rightarrow 0$, we recover the known value for squeezed thermal states 38

$$
\begin{aligned}
g^{(2)} & =\frac{3 \bar{n}_{R}^{2}+2 \bar{n}_{R} \bar{n}_{I}+3 \bar{n}_{I}^{2}}{\left(\bar{n}_{R}+\bar{n}_{I}\right)^{2}} \\
& =2+\frac{(2 \bar{n}+1)^{2} \sinh ^{2} r \cosh ^{2} r}{\left(\bar{n} \cosh (2 r)+\sinh ^{2} r\right)^{2}} \geqslant 2 .
\end{aligned}
$$

Figure 4 shows a density plot of $g^{(2)}$ as a function of $\bar{n}_{R}$ and $\bar{n}_{I}$ for $\left|\alpha_{1}\right|=0.1$ with the maximal allowed value of $w_{1}$, i.e. the bound (32). Again, a whole region of parameter space (delimited by the black dashed curve) corresponds to states displaying antibunching $\left(g^{(2)}<1\right)$, to be contrasted with squeezed thermal states (i.e. without puncture) for which $g^{(2)} \geqslant 2$.

\section{GAUSSIAN-PUNCTURED THERMAL STATES}

We now generalize the results of Sec. III by replacing $\delta$-punctures by single narrow Gaussian punctures. We thus define Gaussian-punctured thermal states as states determined through (1) and (2) with

$$
\begin{aligned}
& P_{\mathrm{cl}}(\alpha)=\frac{e^{-|\alpha|^{2} / \bar{n}}}{\pi \bar{n}}, \\
& \pi(\alpha)=\frac{e^{-|\alpha|^{2} / b}}{\pi b},
\end{aligned}
$$

where $b>0$ characterizes the width of the puncture. Note that $\delta$-punctured thermal states correspond to the limit $b \rightarrow 0$.

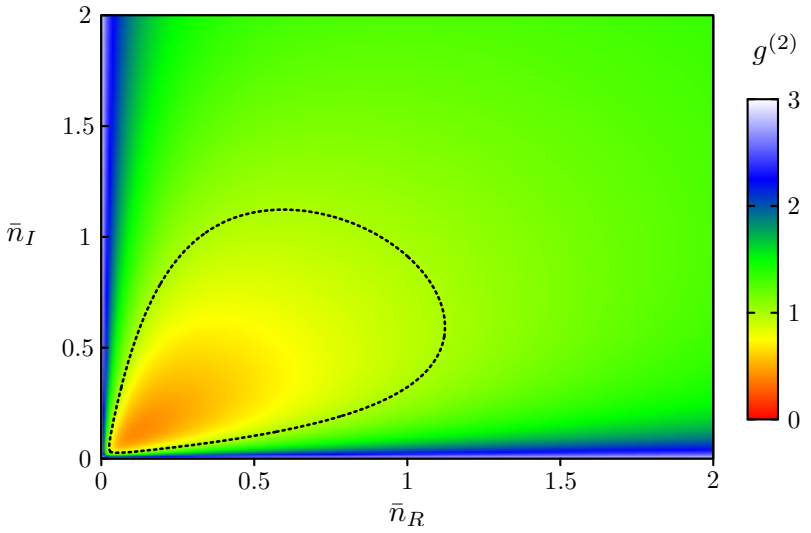

FIG. 4. Density plot of the second order correlation function $g^{(2)}$ [Eq. [39] ] for single $\delta$-punctured squeezed thermal states as a function of $\bar{n}_{R}$ and $\bar{n}_{I}$ for the maximal puncture weight $w_{1}$ given in Eq. 32 for $\alpha_{1 R}=\alpha_{1 I}=0.1 / \sqrt{2}$. The black dashed curve delimits the region where $g^{(2)}<1$. Note that in the absence of puncture, $g^{(2)}>2$ for any squeezed thermal state (see text).

\section{A. Vacuum-centered Gaussian punctures}

We first consider the simple case of a thermal state from which we subtract a single Gaussian centered at $\alpha_{1}=0$. A thermal state with the vacuum component exactly removed (case $b \rightarrow 0, \bar{n}=1, w_{1}=1 / 2$ ) has already been considered in the literature as a simple example of a nonclassical state [40. Here we keep a variable width and amplitude for the subtracted Gaussian.

\section{Condition for (semi)positive definiteness}

Necessary and sufficient condition. Since both the original and the subtracted state are diagonal in the Fock state basis, $\mathcal{N S C}$ can be tackled analytically. The $P$ function of the vacuum-centred Gaussian-punctured thermal state reads

$$
P(\alpha)=\mathcal{N}\left(\frac{e^{-\frac{|\alpha|^{2}}{\bar{n}}}}{\pi \bar{n}}-w_{1} \frac{e^{-\frac{|\alpha|^{2}}{b}}}{\pi b}\right) .
$$

It takes negative values at $\alpha=0$ as soon as $w_{1}>b / \bar{n}$. As we will show, only $b<\bar{n}$ can lead to a positive semidefinite density operator. Expressing the coherent states in the Fock state basis using Eq. (6) and performing the Gaussian integrals, we find

$$
\rho=\mathcal{N} \sum_{n=0}^{\infty}\left[p_{\mathrm{cl}}(n)-w_{1} p_{\pi}(n)\right]|n\rangle\langle n|,
$$

with

$$
p_{\mathrm{cl}}(n)=\frac{\bar{n}^{n}}{(\bar{n}+1)^{n+1}}, \quad p_{\pi}(n)=\frac{b^{n}}{(b+1)^{n+1}} .
$$


Since $\rho$ is diagonal, the positivity condition reads $p_{\mathrm{cl}}(n)-$ $w_{1} p_{\pi}(n) \geqslant 0$ for all $n \in \mathbb{N}_{0}$, which is equivalent to

$$
w_{1} \leqslant \frac{b+1}{\bar{n}+1} \inf _{n \in \mathbb{N}_{0}}\left\{\left(\frac{\bar{n} b+\bar{n}}{\bar{n} b+b}\right)^{n}\right\} \equiv w_{\max , \text { vac }}^{\mathrm{G}, \mathcal{N} \mathcal{S C}} .
$$

We can now distinguish two cases: i) If $b>\bar{n}$, then the fraction in the argument of the infimum is smaller than 1 and the infimum is obtained in the limit $n \rightarrow$ $\infty$ and evaluates to 0 , hence $w_{1}=0$. This shows that we cannot subtract a broader Gaussian than the original one. ii) In the other case, where $b \leqslant \bar{n}$, the minimum is obtained for $n=0$ and evaluates to 1 . We can thus subtract a Gaussian that is tighter than the original one and still obtain a positive semidefinite density operator. This works as long as

$$
w_{1} \leqslant \frac{b+1}{\bar{n}+1} \equiv w_{\max , \mathrm{vac}}^{\mathrm{G}, \mathcal{N} \mathcal{S C}}
$$

\section{Conditions for nonclassicality}

Negativity of the $P$ function. In order to obtain a $P$ function which exhibits negative values for some $\alpha$, we see from Eq. (42) that this requires

$$
w_{1}>\frac{b}{\bar{n}},
$$

showing that in the limit $b \rightarrow 0$, we recover the fact that all $\delta$-punctured states are nonclassical.

Negativity of the Wigner function. The Wigner function of a vacuum-centered Gaussian-punctured thermal state reads

$$
W(\alpha)=\mathcal{N} \frac{2}{\pi}\left(\frac{e^{-\frac{2|\alpha|^{2}}{1+2 \bar{n}}}}{1+2 \bar{n}}-w_{1} \frac{e^{-\frac{2|\alpha|^{2}}{1+2 b}}}{1+2 b}\right),
$$

and takes negative values if $w_{1}$ satisfies

$$
w_{1}>\frac{1+2 b}{1+2 \bar{n}},
$$

which is a stronger condition than Eq. 45). Note that for $b \rightarrow 0$, Eq. (47) tends to Eq. 25), the bound found for $\delta$-punctured thermal states.

Antibunching. From the expression of the second-order correlation function 10$]$, we have

$$
g^{(2)}=\left(1-w_{1}\right) \frac{2 \bar{n}^{2}-w_{1} 2 b^{2}}{\left(\bar{n}-w_{1} b\right)^{2}} .
$$

For $w_{1}=w_{\max , \text { vac }}^{\mathrm{G}, \mathcal{N} \mathcal{S}}$, the condition for antibunching reads

$$
g^{(2)}<1 \quad \Leftrightarrow \quad \bar{n}^{2}+b^{2}<1,
$$

To summarize, for $\alpha_{1}=0$, a $P$ function that corresponds to a positive semidefinite density operator and attains negative values in some region is obtained if and only if

$$
b<\bar{n} \quad \text { and } \quad \frac{b}{\bar{n}}<w_{1} \leqslant \frac{b+1}{\bar{n}+1} .
$$

Hence, the puncture weight $w_{1}$ must be smaller than $(b+1) /(\bar{n}+1)$ to ensure the physicality of the state, but greater than $b / \bar{n}$ to yield a nonclassical state. Moreover, in the limit $b \rightarrow 0$, the Gaussian puncture becomes a $\delta$ puncture, and we recover the value given in (16). This provides an analytical proof of the tightness of the bound $w_{\max }^{\delta, \mathcal{N C}}$ in the case $\alpha_{1}=0$.

\section{B. Arbitrarily centred Gaussian punctures}

We now consider the more general case of an arbitrarily centred Gaussian puncture corresponding to a $P$ function of the form

$$
P(\alpha)=\mathcal{N}\left(\frac{e^{-\frac{|\alpha|^{2}}{\bar{n}}}}{\pi \bar{n}}-w_{1} \frac{e^{-\frac{\left|\alpha-\alpha_{1}\right|^{2}}{b}}}{\pi b}\right) .
$$

We first obtain an upper bound on the amplitude of the puncture, and then support the tightness of this bound with numerical results. Finally, we find the regimes of parameters yielding nonclassicality and antibunching.

\section{Condition for (semi)positive definiteness}

Necessary condition. Using Eq. (7) with $\mathcal{K}$ the set of coherent states, we find that we can only subtract a Gaussian tighter than the original state $(b<\bar{n})$ and obtain as a necessary condition for positivity

$$
w_{1} \leqslant \frac{b+1}{\bar{n}+1} e^{-\frac{\left|\alpha_{1}\right|^{2}}{\bar{n}-b}} \equiv w_{\max }^{\mathrm{G}, \mathcal{N C}} .
$$

Proof. Using $|\langle\gamma \mid \alpha\rangle|^{2}=e^{-|\alpha-\gamma|^{2}}$ and Eq. (41), we find for the expectation value of $\rho$ in the coherent state $|\gamma\rangle$

$$
\rho_{\gamma \gamma}=\int\left(\frac{1}{\pi \bar{n}} e^{-\frac{|\alpha|^{2}}{\bar{n}}-|\alpha-\gamma|^{2}}-\frac{w_{1}}{\pi b} e^{-\frac{\left|\alpha-\alpha_{1}\right|^{2}}{b}-|\alpha-\gamma|^{2}}\right) d^{2} \alpha .
$$

Evaluation of the Gaussian integrals leads to

$$
\rho_{\gamma \gamma} \geqslant 0 \quad \Leftrightarrow \quad w_{1} \leqslant \frac{b+1}{\bar{n}+1} e^{-\frac{|\gamma|^{2}}{\bar{n}+1}+\frac{\left|\gamma-\alpha_{1}\right|^{2}}{b+1}} .
$$

That needs to be true for all $\gamma \in \mathbb{C}$ in order to keep the possibility of a positive semidefinite density operator. Minimizing this expression over $\gamma$ gives us the upper bound $w_{\max }^{\mathrm{G}, \mathcal{N C}}$. Since the exponential function is strictly monotonically increasing and the prefactors are positive we can focus on minimizing the exponent:

$$
f(\gamma) \equiv-\frac{|\gamma|^{2}}{\bar{n}+1}+\frac{\left|\gamma-\alpha_{1}\right|^{2}}{b+1}
$$


By applying a rotation in phase-space, the puncture's center can always be brought along the real axis, so that we can set $\alpha_{1}=x_{1} \in \mathbb{R}$. We then split $\gamma$ into its real and imaginary part $\gamma=\gamma_{R}+i \gamma_{I}$, leading to

$$
f(\gamma)=h\left(\gamma_{I}\right)+g\left(\gamma_{R}\right)+\frac{x_{1}^{2}}{b+1}
$$

with

$$
\begin{aligned}
& h\left(\gamma_{I}\right)=\gamma_{I}^{2}\left(\frac{1}{b+1}-\frac{1}{\bar{n}+1}\right), \\
& g\left(\gamma_{R}\right)=\gamma_{R}^{2}\left(\frac{1}{b+1}-\frac{1}{\bar{n}+1}\right)-\frac{2 \gamma_{R} x_{1}}{b+1} .
\end{aligned}
$$

Here we have to distinguish two cases (the case $b=\bar{n}$ is trivial): i) If $b>\bar{n}, h\left(\gamma_{I}\right)$ is an inverted parabola and can attain arbitrary negative values and the same holds for $f$, thus pushing the exponential in (54) arbitrary close to 0 , implying that the above condition can only be fulfilled for all coherent states if $w_{1}=0$. This means that we cannot subtract any Gaussian wider than the original thermal one and still obtain a valid state. This generalizes what we have already seen in the case of the vacuum centred Gaussian. ii) For $b<\bar{n}$, the dependence of $f$ on the parameters $\gamma_{R}, \gamma_{I}$ splits into the two functions $h$ and $g$ that depend on different independent arguments $\gamma_{R}$ and $\gamma_{I}$. Thus we can minimize both $h$ and $g$ separately. Obviously $h$ is minimized for $\gamma_{I}=0$. We find that $g\left(\gamma_{R}\right)$ is minimized for $\gamma_{R \text {, min }}=(\bar{n}+1) x_{1} /(\bar{n}-b)$ and we get the minimal values

$$
\begin{aligned}
& g\left(\gamma_{R, \text { min }}\right)=-x_{1}^{2} \frac{\bar{n}+1}{b+1} \frac{1}{\bar{n}-b}, \\
& f\left(\gamma_{R, \text { min }}\right)=-\frac{x_{1}^{2}}{\bar{n}-b} .
\end{aligned}
$$

Finally, for the general case $\alpha_{1} \in \mathbb{C}$, plugging $f\left(\gamma_{R, \text { min }}\right)$ into Eq. (54) yields the bound (52).

Tightness of $w_{\max }^{G, \mathcal{N C}}$. As for the previous bounds obtained from the necessary condition (7), diagonalizing the density operator of the Gaussian punctured thermal state [i.e. Eq. (1) together with Eqs. (2) and (41)] and using the $\mathcal{N S C}$ suggest that the bound $w_{\max }^{\mathrm{G}, \mathcal{N C}}[\mathrm{Eq}$. [52]] is tight. This result is shown in Fig. 55, where we plotted the analytic bound (lines) and the numerical bound (squares and dots) for different cut-off dimensions $n_{\max }$ as a function of the center of the puncture $\left|\alpha_{1}\right|$. Also, we found that the coherent state $|\gamma\rangle$ with $\gamma=(\bar{n}+1) /(\bar{n}-b) \alpha_{1}$ is an eigenstate of $\rho$ with eigenvalue 0 when $w_{1}$ is given by the bound $(52)$.

\section{Conditions for nonclassicality}

Negativity of the $P$ function. From Eq. (51), we see that partly negative $P$ functions can be obtained when

$$
w_{1}>\frac{b}{\bar{n}} e^{-\frac{\left|\alpha_{1}\right|^{2}}{\bar{n}-b}},
$$

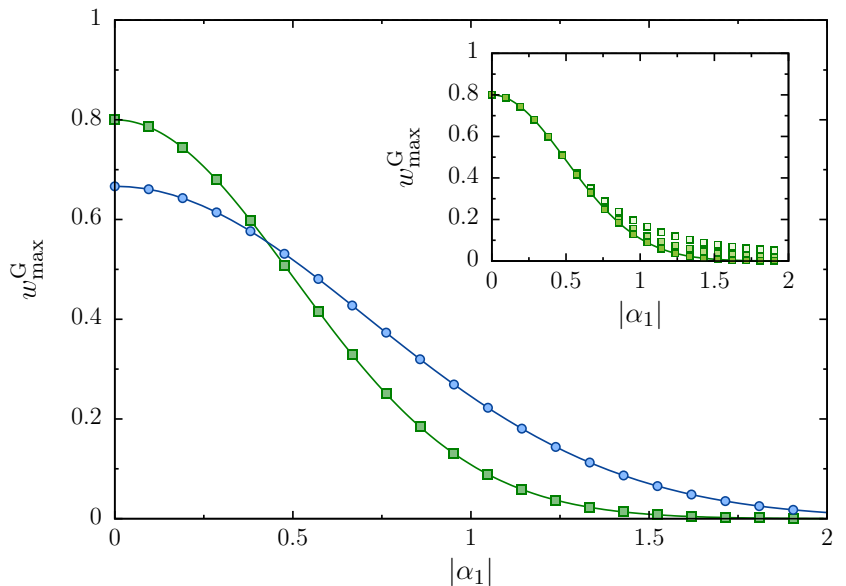

FIG. 5. Plot of the upper bound $w_{\max }^{\mathrm{G}, \mathcal{N C}}$ (solid curve) and of $w_{\max }^{\mathrm{G}}$, the $w_{1}$ which cancels the smallest eigenvalue of the density matrix, as a function of $\left|\alpha_{1}\right|$ for $b=1$ and $\bar{n}=1.5$ (squares) and $\bar{n}=2$ (dots). The eigenvalues are computed in a Fock space of dimension $n_{\max }=40$. Inset: convergence of $w_{\max }^{\mathrm{G}}$ (green squares) as a function of the cut-off dimension $n_{\max }\left(n_{\max }=8,12,40\right.$ from top to bottom $)$.

which generalizes Eq. 45). Hence, the lower bound of the weight decreases with the distance between the center of the thermal state and the position of the puncture. Further this is smaller than $w_{\max }^{\mathrm{G}, \mathcal{N C}}$ given in 52 since $\bar{n}>b$, implying that for all allowed values of $\alpha_{1}, \bar{n}$ and $b$, there is a puncture weight such that the $P$ function is negative somewhere in the complex plane, and the state is nonclassical.

Negativity of the Wigner function. The Wigner function of an arbitrarily-centred Gaussian-punctured thermal state reads

$$
W(\alpha)=\frac{2 \mathcal{N}}{\pi}\left(\frac{e^{-\frac{2|\alpha|^{2}}{1+2 \bar{n}}}}{1+2 \bar{n}}-w_{1} \frac{e^{-\frac{2\left|\alpha-\alpha_{1}\right|^{2}}{1+2 b}}}{1+2 b}\right),
$$

and is negative if $w_{1}$ satisfies

$$
w_{1}>\frac{1+2 b}{1+2 \bar{n}} e^{-\frac{\left|\alpha_{1}\right|^{2}}{\bar{n}-b}},
$$

a stronger condition than Eq. (55).

Antibunching. The correlation function 10 for the state (51) is given by

$$
g^{(2)}=\left(1-w_{1}\right) \frac{2 \bar{n}^{2}-w_{1}\left(\left|\alpha_{1}\right|^{4}+4\left|\alpha_{1}\right|^{2} b+2 b^{2}\right)}{\left[\bar{n}-w_{1}\left(\left|\alpha_{1}\right|^{2}+b\right)\right]^{2}} .
$$

Note that in the limit $b \rightarrow 0$, Eq. (58) tends to Eq. (26) found in Sec. III for a $\delta$-punctured thermal state. We have that for $\bar{n}<1$, the minimum of $g^{(2)}$ is obtained for $b \rightarrow 0$. For $\bar{n} \rightarrow 0$ this minimum tends to 0 . We also have that for $\bar{n}>1$, the minimum of $g^{(2)}$ is obtained for a finite value of $b$. Figure 6 shows Eq. (58) as a function of $\bar{n}$ and $b<\bar{n}$ for $\alpha_{1}=0.1$ and for the maximal puncture weight $w_{1}=w_{\max }^{\mathrm{G}, \mathcal{N C}}$ given in Eq. (52). 


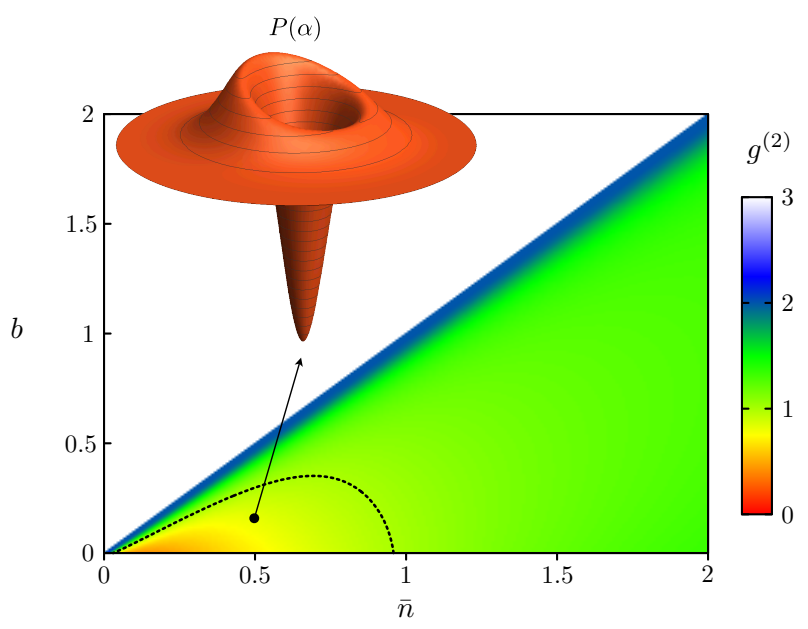

FIG. 6. Density plot of the second order correlation function $g^{(2)}$ as a function of $\bar{n}$ and $b<\bar{n}$ for single Gaussian-punctured states with $\alpha_{1}=0.1$ for the maximal puncture weight $w_{1}$ given in Eq. (52). The black dashed curve delimits the region where $g^{(2)}<1$. In the top left corner is shown the $P$ function for the parameters indicated by a dot in the density plot.

\section{POSSIBLE EXPERIMENTAL REALIZATIONS OF PUNCTURED STATES}

\section{A. Vacuum-removed states}

A $\delta$-punctured thermal state with puncture at $\alpha_{1}=0$ and maximum weight of the puncture, $b_{\mathcal{N S \mathcal { C }}}^{\text {gaus,vac }}$, i.e. a vacuum-removed thermal state,

$\rho=\mathcal{N} \sum_{n=1}^{\infty} \frac{\bar{n}^{n}}{(1+\bar{n})^{n+1}}|n\rangle\langle n|=\frac{\bar{n}+1}{\bar{n}}\left[\rho_{T}-\frac{1}{1+\bar{n}}|0\rangle\langle 0|\right]$

is probably the example of a punctured state that is easiest to realize experimentally. Since a thermal state has no coherences between Fock states, a projective measurement in the Fock-basis does not destroy the quantum properties of the state, but only alters its statistical character. Discarding the ground-state when it is found leads to an ensemble of states that realizes the vacuum-removed thermal state. This generalizes to any initial state that is diagonal in the Fock-basis. When single-photon sources are available, one can of course synthesize a given vacuum-removed state diagonal in the Fock basis from the beginning by mixing Fock-states with the corresponding statistical weight without the need for any measurement. This generalizes to states where the vacuum is not removed completely by adjusting the statistical weight of the vacuum-state to the desired value.

The measurement-based procedure faces the problem that traditional photon-counting methods destroy the photons during the measurement. Here we propose a procedure suitable for cavity-QED or circuit-QED that realizes a vacuum-removed state diagonal in the Fock basis, based on the nondestructive detection of photons [41-45] in a mode of the cavity (See Fig. 7). Two-level atoms in the cavity in a superposition $|+\rangle$ of their two states $|g\rangle,|e\rangle$, where $| \pm\rangle=(|g\rangle \pm|e\rangle) / \sqrt{2}$, experience a phase shift $\varphi=\frac{\pi(n \bmod 2 q)}{q}$ between $|g\rangle$ and $|e\rangle$ that depends on the number of photons in the cavity, with an integer $q$ that can be controlled through the interaction time and strength. A projective measurement of an atom in the basis $\{| \pm\rangle\}$ (corresponding to measurements results labelled \pm ) updates our knowledge of the photon number. Since $\varphi$ is defined modulo $2 \pi, 2 q$ different Fock states can be distinguished.

However, we need no information which Fock state is actually realized, but only need to systematically exclude the vacuum state. This can be achieved with an iterative procedure: In the first step one sets $q=1=2^{0}$. If ' - ' is measured, the state is tagged as part of the ensemble. If ' + ' is measured, we still need to distinguish the states $|n\rangle$ with $n=0,2,4,6,8, \ldots$ in order to not truncate more states than the vacuum. So we set $q=2=2^{1}$ yielding a phase shift of $\varphi=\frac{\pi(n \bmod 4)}{2}$ that allows us to distinguish states with $n=2+4 k, k \in \mathbb{N}_{0}$ photons (measurement result "-") from states with $n=4 k, k \in \mathbb{N}_{0}$, including the vacuum (measurement result "+"). This can be continued with $q=2^{l-1}, l=3,4, \ldots, l_{\max }$, as long as needed to exclude the ambiguity of states with even photon numbers as high as wished.

In general, setting $q=2^{l-1}$, with $l \in \mathbb{N}$, we can definitely distinguish the vacuum from all states with $n=q+2 k q, k \in \mathbb{N}_{0}$. If after a finite number of repetitions $l_{\text {max }}$ all measurements yielded ' $+{ }^{\prime}$ we need to completely discard the remaining state. This obviously causes some errors, since we also exclude possible realizations other than the vacuum from being further used. The smallest photon number that was not distinguished from the vacuum, and was therefore also subtracted in the remaining state, is then $n_{0}=2 q_{\max }=2^{l_{\max }}$, where $q_{\max }$ corresponds to the smallest interaction time that has been realized, i.e. the last measurement. The whole procedure post-selects states $|n\rangle \neq\left|2^{l_{\max }} k\right\rangle \forall k \in \mathbb{N}_{0}$, whereas states $|n\rangle=\left|2^{l_{\max }} k\right\rangle, k \in \mathbb{N}_{0}$ are truncated. The procedure only works because the thermal state is diagonal in the Fock-basis; all coherences between different Fock states are lost. In such a case, however, the procedure is very effective: If we want to make sure that states with photon number less than $n_{0}$ were not truncated, we only need to perform $l_{\max }=\left\lceil\log _{2}\left(n_{0}\right)\right\rceil$ different measurements.

For a thermal state, occupation for high photon number states decays very rapidly for small temperatures, and the overall error can be kept small. To quantify this, we can calculate the fidelity of our desired state 59 with 


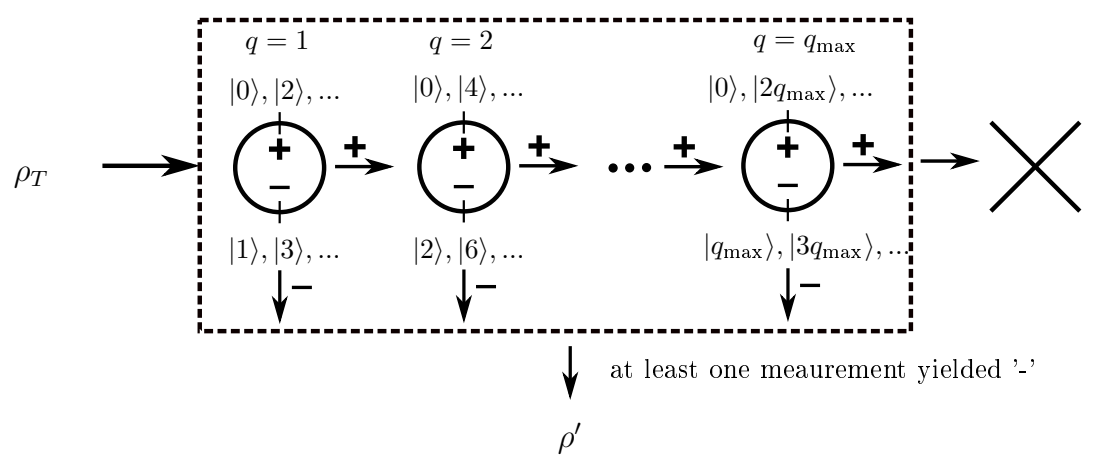

FIG. 7. Sketch of realization of vacuum-removed thermal state. A thermal state $\rho_{T}$ is subjected to multiple QND-measurements that distinguish the vacuum state of other photon number states. If at least one of the measurements yields ' $-^{\prime}$, the vacuum has definitely not been realized and the measured state is added to the ensemble. If all measurements yielded ' $+{ }^{\prime}$ the state is discarded. Since we only perform a finite number of measurements, the final state $\rho^{\prime}$ given in equation 60 can slightly deviate from the desired state $\rho$ given in equation (59). The deviation can be quantified calculating the fidelity (61).

respect to the state that was actually constructed

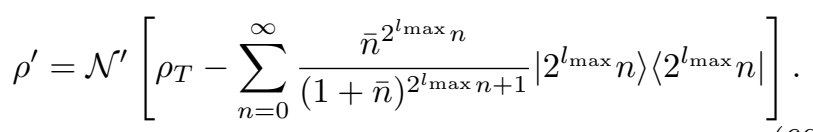

Since both density operators are diagonal in Fock basis the fidelity is simply evaluated as [46, p. 409]:

$$
F\left(\rho, \rho^{\prime}\right)=\sum_{n=0}^{\infty} \sqrt{\rho_{n n} \rho_{n n}^{\prime}}=1-\frac{1}{\bar{n}} \frac{1}{\left(\frac{\bar{n}+1}{\bar{n}}\right)^{2^{l_{\max }}}-1} .
$$

In the limit of large $l_{\max }$ this reduces to

$$
F=1-\frac{1}{\bar{n}}\left(\frac{\bar{n}}{\bar{n}+1}\right)^{2^{l_{\max }}}=1-\frac{e^{-\log \left(\frac{\bar{n}+1}{\bar{n}}\right) 2^{l_{\max }}}}{\bar{n}},
$$

i.e. the fidelity approaches 1 exponentially with $2^{l_{\max }}$.

This shows the theoretical viability of the procedure. Experimental imperfections, as for example the non perfect linearity of the phase shift in the photon numbers [43, may worsen the result and will have to be evaluated for a given experimental setup.

\section{B. "Vacuum or not"-measurements}

The vacuum-state can be removed from a broader class of states, namely states that do not contain coherences between the vacuum state and other Fock states. Indeed, if $\rho_{n n}=0$ in the Fock basis, then $\rho_{0 n}=\rho_{n 0}=0$ is implied by the positivity of the state [47]. Then one can remove the vacuum-state with a measurement operator $A$ of the form

$$
A=a_{0}|0\rangle\left\langle 0\left|+a_{1} \sum_{n=1}^{\infty}\right| n\right\rangle\langle n| .
$$

with $a_{0} \neq a_{1}$ : When $a_{0}$ is measured one discards the state, for $a_{1}$ one continues the experiment and effectively gets the desired punctured state through post-selection. Recently a procedure was proposed that realizes the measurement operator $A$ [48].

\section{Synthesizing arbitrary states}

The above considerations are only valid for a complete subtraction of the vacuum state. The procedure does not translate easily to more general subtractions, especially for $\alpha_{1} \neq 0$, and we therefore present another, completely different approach, based on approximately synthesizing our states from scratch as follows: We can numerically express the density operator of the desired punctured state up to a chosen dimension $N$ in the Fock basis, neglecting terms involving states with higher photon number than $N$ :

$$
\rho^{\prime}=\sum_{n=0}^{N} \sum_{m=0}^{N} c_{m n}|n\rangle\langle m| .
$$

This is in general a mixed state and we can express it as a sum of pure states:

$$
\begin{gathered}
\rho^{\prime}=\sum_{i=1} p_{i}\left|\psi_{i}\right\rangle\left\langle\psi_{i}\right| \\
\left|\psi_{i}\right\rangle=\sum_{n=0}^{N} c_{n}^{(i)}|n\rangle .
\end{gathered}
$$

We can then create our state by choosing with the right probability one of the pure states and constructing it with the method proposed in [49 52] and demonstrated experimentally in [53]. Multiple repetition (creation of an adequate ensemble of states) then gives us our state up to a certain accuracy. In a single run we actually do not have the complete state, but for further experiments one anyway needs typically multiple repetitions for obtaining 
measurement statistics of any observable. Hence, this procedure creates the desired mixed state in the ensemble sense.

\section{CONCLUSION}

In this work, we introduced a novel class of nonclassical states with regular non-positive Glauber-Sudarshan $P$ function that we call punctured states. These states are obtained from the addition of sufficiently narrow negative peaks to smooth positive $P$ functions. We determined the regimes of parameters yielding proper physical (i.e. positive semidefinite) nonclassical states in the case of $\delta$ - and Gaussian punctures of thermal or squeezed thermal states. We showed that their second-order corre- lation function can be modified through an appropriate choice of the punctures and identify the regimes yielding antibunching of light. All states exhibiting antibunching have a negative $P$ function in some region. Finally, we presented possible experimental realizations of punctured states based on vacuum-or-not measurements and on complete synthesizing.

\section{ACKNOWLEDGMENTS}

FD acknowledges fruitful discussions with Daniel K. L. Oi. FD would like to thank the F.R.S.-FNRS for financial support. Computational resources have been provided by the Consortium des Équipements de Calcul Intensif (CÉCI), funded by the F.R.S.-FNRS under Grant No. 2.5020.11.
[1] D. V. Strekalov and G. Leuchs, Nonlinear interactions and nonclassical light, in Advances in Quantum Photonics: from the First Single-photon and Nonlinear Optical Experiments to Modern Quantum Photon$i c s$, edited by Robert W. Boyd, Svetlana G. Lukishova, and Victor N. Zadkov (Springer, New York, in press), arXiv:1701.01403.

[2] A. Pathak and A. Ghatak, Classical light vs. nonclassical light: characterizations and interesting applications, Journal of Electromagnetic Waves and Applications 32, 229 (2018).

[3] M. Xiao, L.-A. Wu, and H. J. Kimble, Precision measurement beyond the shot-noise limit, Phys. Rev. Lett. 59, 278 (1987).

[4] L. K. Shalm, R. B. A. Adamson, and A. M. Steinberg, Squeezing and over-squeezing of triphotons, Nature (London) 457, 67 (2009).

[5] T. Ono, J. Sabines-Chesterking, H. Cable, J. L. O'Brien, and J. C. F. Matthews, Optical implementation of spin squeezing, New J. Phys. 19, 053005 (2017).

[6] A. N. Boto, P. Kok, D. S. Abrams, S. L. Braunstein, C. P. Williams, and J. P. Dowling, Quantum interferometric optical lithography - Exploiting entanglement to beat the diffraction limit, Phys. Rev. Lett. 85, 2733 (2000).

[7] D. V. Strekalov and J. P. Dowling, Two-Photon Interferometry for High-Resolution Imaging, J. Mod. Opt. 49, 519 (2002).

[8] M. Genovese, Real applications of quantum imaging, J. Opt. 18, 073002 (2016).

[9] M. A. Taylor, J. Janousek, V. Daria, J. Knittel, B. Hage, H.-A. Bachor, and Warwick P. Bowen, SubdiffractionLimited Quantum Imaging within a Living Cell, Phys. Rev. X 4, 011017 (2014).

[10] B. Ya. Zel'dovich and D. N. Klyshko, Statistics of field in parametric luminescence, Pis'ma Zh. Eksp. Teor. Fiz. 9, 69 (1969) [JETP Lett. 9, 40 (1969)].

[11] D. C. Burnham and D. L. Weinberg, Observation of simultaneity in parametric production of optical photon pairs, Phys. Rev. Lett. 25, 84 (1970).

[12] D. N. Klyshko, Use of two-photon light for absolute calibration of photoelectric detectors, Sov. J. Quantum Elec- tron. 10, 1112 (1980).

[13] G. Brassard, N. Lütkenhaus, T. Mor, and B. C. Sanders, Limitations on practical quantum cryptography, Phys. Rev. Lett. 85, 1330 (2000).

[14] H. Zheng, D. J. Gauthier, and H. U. Baranger, Decoystate quantum key distribution with nonclassical light generated in a one-dimensional waveguide, Opt. Lett. 38, 622 (2013).

[15] S. D. Bartlett, B. C. Sanders, Requirement for quantum computation, J. Mod. Opt. 50, 2331 (2003).

[16] R. Chiao and J. Garrison, Quantum optics (Oxford University Press, 2008).

[17] E. C. G. Sudarshan, Equivalence of Semiclassical and Quantum Mechanical Descriptions of Statistical Light Beams, Phys. Rev. Lett. 10, 277 (1963).

[18] G. S. Agarwal, Quantum Optics (Cambridge University Press, 2012).

[19] T. Kiesel and W. Vogel, Nonclassicality filters and quasiprobabilities, Phys. Rev. A 82, 032107 (2010).

[20] T. Kiesel, W. Vogel, B. Hage, and R. Schnabel, Direct sampling of negative quasiprobabilities of a squeezed state, Phys. Rev. Lett. 107, 113604 (2011).

[21] E. Agudelo, J. Sperling, and W. Vogel, Quasiprobabilities for multipartite quantum correlations of light, Phys. Rev. A 87, 033811 (2013).

[22] B. Kühn and W. Vogel, Visualizing nonclasscal effects in phase space, Phys. Rev. A 90, 033821 (2014).

[23] D. N. Klyshko, Observable signs of nonclassical light, Phys. Lett. A 213, 7 (1996).

[24] A. Brauer, Limits of the characteristic roots of a matrix, Duke Mathematical Journal 13, 387 (1946).

[25] P. Shivakumar, J. Williams, and N. Rudraiah, Eigenvalues for infinite matrices, Linear Algebra and its Applications 96, 35 (1987).

[26] D. T. Smithey, M. Beck, M. G. Raymer, and A. Faridani, Measurement of the Wigner distribution and the density matrix of a light mode using optical homodyne tomography: Application to squeezed states and the vacuum, Phys. Rev. Lett. 70, 1244 (1993).

[27] S. Wallentowitz and W. Vogel, Unbalanced homodyning for quantum-state measurements, Phys. Rev. A 53, 4528 
(1996).

[28] K. Banaszek and K. Wódkiewicz, Direct probing of quantum phase space by photon counting, Phys. Rev. Lett. 76, 4344 (1996).

[29] L. G. Lutterbach and L. Davidovich, Method for direct measurement of the Wigner function in cavity $Q E D$ and ion traps, Phys. Rev. Lett. 78, 2547 (1997).

[30] K. Banaszek, C. Radzewicz, K. Wódkiewicz, and J. S. Krasiński, Direct measurement of the Wigner function by photon counting, Phys. Rev. A 60, 674 (1999).

[31] P. Bertet, A. Auffeves, P. Maioli, S. Osnaghi, T. Meunier, M. Brune, J. M. Raimond, and S. Haroche, Direct Measurement of the Wigner Function of a One-Photon Fock State in a Cavity, Phys. Rev. Lett. 89, 200402 (2002).

[32] W. P. Schleich, "Quantum Optics in Phase space", WileyVCH Verlag Berlin GmbH, Berlin (2001).

[33] L. Mandel, Sub-Poissonian photon statistics in resonance fluorescence, Opt. Lett. 4, 205 (1979).

[34] H. J. Kimble, M. Dagenais, and L. Mandel, Photon Antibunching in Resonance Fluorescence, Phys. Rev. Lett. 39, 691 (1977).

[35] W. Vogel, Nonclassical correlation properties of radiation fields, Phys. Rev. Lett. 100, 013605 (2008).

[36] J. L. W. V. Jensen, Sur les fonctions convexes et les inégalités entre les valeurs moyennes, Acta Math. 30, 175 (1906).

[37] M. O. Scully and M. S. Zubairy, Quantum Optics (Cambridge University Press, 2001).

[38] M. S. Kim, F. A. M. de Oliveira, and P. L. Knight, Properties of squeezed number states and squeezed thermal states, Phys. Rev. A 40, 2494 (1989).

[39] S. M. Barnett and P. M. Radmore, Methods in Theoretical Quantum Optics (Oxford University Press, Oxford, 2002).

[40] L. Diósi, Comment on "Nonclassical States: An Observable Criterion", Phys. Rev. Lett. 85, 2841 (2000).

[41] G. Gabrielse, S. Peil, B. Odom, and B. D'Urso, QND observation of quantum jumps between Fock states: a oneelectron cyclotron oscillator at $70 \mathrm{mK}$ to $4.2 \mathrm{~K}$, in Summaries of Papers Presented at the Quantum Electronics and Laser Science Conference, 1999. QELS'99. Technical Digest. (IEEE,1992) pp. 19-20.

[42] A. Reiserer, S. Ritter, and G. Rempe, Non-destructive detection of an optical photon, Science 342, 1349 (2013).
[43] C. Guerlin, J. Bernu, S. Deleglise, C. Sayrin, S. Gleyzes, S. Kuhr, M. Brune, J.-M. Raimond, and S. Haroche, Progressive field-state collapse and quantum non-demolition photon counting, Nature (London) 448, 889 (2007).

[44] Y. F. Xiao, S. K. Özdemir, V. Gaddam, C. H. Dong, N. Imoto and L. Yang, Quantum nondemolition measurement of photon number via optical Kerr effect in an ultrahigh-Q microtoroid cavity, Opt. Exp. 16, 21462 (2008).

[45] F. Helmer, M. Mariantoni, E. Solano, and F. Marquardt, Quantum nondemolition photon detection in circuit $Q E D$ and the quantum Zeno effect, Phys. Rev. A 79, 052115 (2009).

[46] M. A. Nielsen and I. L. Chuang, Quantum computation and quantum information (Cambridge University Press, 2010).

[47] R. A. Horn and C. R. Johnson, Matrix Analysis (Cambridge University Press, New York, 1985).

[48] D. K. L. Oi, V. Potoček, and J. Jeffers, Nondemolition Measurement of the Vacuum State or its Complement, Phys. Rev. Lett. 110, 210504 (2013).

[49] M. Hofheinz, E. M. Weig, M. Ansmann, R. C. Bialczak, E. Lucero, M. Neeley, A. D. O'Connell, H. Wang, J. M. Martinis, and A. N. Cleland, Generation of Fock states in a superconducting quantum circuit, Nature (London) 454, 310 (2008).

[50] A. Ben-Kish, B. DeMarco, V. Meyer, M. Rowe, J. Britton, W. M. Itano, B. M. Jelenković, C. Langer, D. Leibfried, T. Rosenband, and D. J. Wineland, Experimental Demonstration of a Technique to Generate Arbitrary Quantum Superposition States of a Harmonically Bound Spin-1/2 Particle, Phys. Rev. Lett. 90, 037902 (2003).

[51] C. K. Law and J. H. Eberly, Arbitrary Control of a Quantum Electromagnetic Field, Phys. Rev. Lett. 76, 1055 (1996)

[52] Y.-X. Liu, L.-F. Wei, and F. Nori, Generation of nonclassical photon states using a superconducting qubit in a quantum electrodynamic microcavity, Europhys. Lett. 67, 941 (2004)

[53] M. Hofheinz, H. Wang, M. Ansmann, R. C. Bialczak, E. Lucero, M. Neeley, A. O'Connell, D. Sank, J. Wenner, J. M. Martinis, and A. N. Cleland, Synthesizing arbitrary quantum states in a superconducting resonator, Nature (London) 459, 546 (2009). 